\title{
Modelagem numérica comparativa da ponte Florestinha, construída em madeira e concreto
}

\author{
Comparative numerical modelling of the Florestinha \\ bridge, built in a timber and concrete composite \\ structure
}

\section{Leonor da Cunha Mastela \\ Pedro Gutemberg de Alcântara Segundinho \\ Vinicius Borges Taquetti \\ André Luis Christoforo \\ Francisco Antonio Rocco Lahr \\ Tiago Aguiar Gales \\ Resumo}

${ }^{1}$ Leonor da Cunha Mastela 'Universidade Federal do Espírito Santo Jerônimo Monteiro - ES - Brasil

${ }^{2}$ Pedro Gutember de Alcântara Segundinho 2Universidade Federal do Espírito Santo Jerônimo Monteiro - ES - Brasil

${ }^{3}$ Vinicius Borges Taquetti ${ }^{3}$ Universidade Federal do Espírito Santo Jerônimo Monteiro - ES - Brasil

${ }^{4}$ André Luis Christoforo ${ }^{4}$ Universidade Federal de São Carlos São Carlos - SP - Brasil

${ }^{5}$ Francisco Antonio Rocco Lahr 5Universidade Federal de São Carlos São Carlos - SP - Brasil

${ }^{6}$ Tiago Aguiar Gales ${ }^{6}$ Universidade Federal do Espírito Santo Jerônimo Monteiro - ES - Brasil

Recebido em 13/11/19 Aceito em 04/01/21
A

possibilidade de executar estruturas com elementos que apresentam seções mistas de materiais como madeira e concreto traz benefícios, como o aumento da economia na construção e da durabilidade à abrasão. O objetivo do trabalho consiste em realizar a comparação dos resultados de deslocamento vertical obtidos em modelagem numérica utilizando-se o método dos elementos finitos, resultados teóricos encontrados pelo programa Orthotropic Timber Bridges e experimentais da ponte Florestinha. Foram realizados três modelos numéricos. Considerou-se para os modelos 1 e 2 que as vigas de madeira são roliças e, para o modelo 3, que as vigas são de seção quadrada. Com os resultados obtidos, constatou-se que o modelo 1 se aproxima dos dados experimentais e apresenta desvio padrão do erro para o deslocamento devido ao carregamento central de $\pm 0,69 \mathrm{~mm}$, que corresponde a $1,56 \%$ do erro percentual médio, e para o carregamento lateral de $\pm 0,73 \mathrm{~mm}$, que indica $1,12 \%$ do erro percentual médio. Os modelos numéricos 1 e 2 representaram satisfatoriamente os modelos experimentais, revelando que a aplicação do método dos elementos finitos foi satisfatória para a análise da ponte.

Palavras-chave: Método dos elementos finitos. Deslocamento vertical. Estruturas mistas. Construções rurais.

\section{Abstract}

Composite structures built with sections made of wood and concrete are more durable and economic. The performance of these structures can be analysed with programmes that use the finite element method. The purpose of this study is to compare the vertical displacement obtained by the finite element method, the theoretical results found by the Orthotropic Timber Bridges programme and an experimental analysis of the Florestinha bridge. Three numerical models were performed. For models 1 and 2, timber beams with circular cross sections were applied, and for model 3, beams with a square cross section were used. The results of model 1 are close to the experimental data and present standard deviation for displacement under the central loading of \pm $0.69 \mathrm{~mm}$, which is $1.56 \%$ of the mean percentage error, and presents \pm 0.73 $\mathrm{mm}$ for the lateral loading, which is $1.12 \%$ of the mean percentage error. The numerical models 1 and 2 satisfactorily represented the experimental models, demonstrating that the application of the finite element method was satisfactory for the analysis of the bridge in question.

Keywords: Finite element method. Vertical displacement. Composite structures. Rural constructions.

MASTELA, L. da C.; SEGUNDINHO, P. G. de A.; TAQUETTI, V. B.; CHRISTOFORO, A. L.; LAHR, F. A. R.; GALES, T. A. Modelagem numérica comparativa da ponte Florestinha construída em madeira e concreto. Ambiente Construído, Porto Alegre, v. 21, n. 3, p. 295-304, jul./set. 2021. 


\section{Introdução}

A utilização de estruturas mistas, com elementos de seções compostas de madeira e concreto, representa uma solução viável em construções rurais, pois pode apresentar bom desempenho estrutural e econômico, além de durabilidade satisfatória (CALIL JUNIOR; DIAS, 1997; YEOH et al., 2011; RODRIGUES; DIAS; PROVIDÊNCIA, 2013). A utilização combinada de madeira e concreto garante maior rigidez e resistência em relação à estrutura executada apenas em madeira (MOLINA; CALIL JUNIOR, 2009; DIAS et al., 2015).

$\mathrm{Na}$ engenharia estrutural, o método dos elementos finitos (MEF) permite simular a deformação de elementos sólidos e o estado de tensão de quaisquer tipos de geometria, sendo estes submetidos a ações externas, o que pode garantir confiabilidade e simplicidade na implementação computacional (AZEVEDO, 2003). Basicamente, o MEF pode ser definido como um método matemático no qual um meio contínuo é discretizado em elementos que representam as propriedades originais do sólido por meio de equações diferenciais (LOTTI et al., 2006).

No MEF, a estrutura é decomposta em pequenos elementos, denominados subdomínios. No interior do elemento finito, um campo com características elásticas se manifesta como um agrupamento linear de um número finito de campos elementares com deformabilidade (SEGUNDINHO, 2005). A modelagem da estrutura pode ser elaborada mediante a discretização dos materiais que integram a seção mista (OUDJENE et al., 2013). O MEF pode ser empregado para o estudo do desempenho de vigas mistas de madeira e concreto, permitindo comparar os resultados obtidos experimental e computacionalmente, o que possibilita a verificação do grau de interação existente entre a mesa de concreto e a alma de madeira que compõem a estrutura (MASCIA et al., 2013; MOLINA; CALIL JUNIOR, 2018).

Muitos estudos têm proposto modelos numéricos para a simulação de estudos experimentais de estruturas mistas de madeira e concreto. Frangiacomo (2005) aplicou o MEF no estudo de vigas compostas de madeira e concreto sob carga de longo prazo. Para a modelagem, foram considerados a deformabilidade do sistema de conectores e o comportamento reológico do concreto e da madeira. A possibilidade de prever com precisão a resposta no longo prazo foi mostrada comparando-se resultados numéricos e experimentais para diferentes situações. Concluiu-se que o modelo sugerido é adequado para a análise de vigas mistas de madeira e concreto submetidas aos carregamentos propostos no estudo.

Zona, Barbato e Fragiacomo (2012) utilizaram modelagem numérica não linear pelo MEF para simular a conexão de cisalhamento deformável de vigas compostas de madeira e concreto, para estimar a resposta estrutural de curto prazo para a qual existem resultados experimentais. O estudo teve como objetivo analisar a correlação entre avaliações experimentais e resultados numéricos com base em modelos do MEF. Os resultados obtidos mostraram que ainda são necessários estudos adicionais relacionados aos parâmetros constitutivos dos conectores de madeira e concreto, já que estes influenciam significativamente a correlação entre os resultados experimentais e os numéricos.

O estudo de Monteiro, Dias e Lopes (2015) comparou alguns modelos teóricos com resultados experimentais para lajes mistas de madeira e concreto sob aplicação de cargas concentradas. $\mathrm{O}$ trabalho analisou em seus modelos deslocamentos verticais e distribuição das reações de apoio das vigas de madeira. A comparação entre os modelos e os resultados experimentais permitiu a validação dos modelos simulados por apresentar resultados suficientemente próximos. O estudo concluiu que modelos numéricos para avaliar a distribuição transversal de carga podem ser empregados na prática de projeto.

O presente trabalho tem como objetivo comparar os resultados dos modelos para os deslocamentos verticais originários da simulação numérica utilizando o MEF, por meio do software Structural Analysis Program (SAP2000) (COMPUTERS..., 2012) estudante, com os resultados experimentais da ponte Florestinha.

\section{Material e métodos}

A pesquisa tem como base as informações e os resultados descritos por Pigozzo (2004) referentes ao projeto, à construção e aos ensaios da ponte Florestinha. O estudo de Pigozzo (2004) consiste na aplicação de barras de aço coladas como conectores em elementos mistos de madeira e concreto para tabuleiros de pontes. Na Figura 1, pode-se visualizar a ponte Florestinha.

A ponte Florestinha está localizada na estrada municipal que faz ligação entre o município de Piracicaba e o de Anhambi, SP, com coordenadas geográficas $22^{\circ} 45^{\prime} 40^{\prime \prime} \mathrm{S}$ e $47^{\circ} 45^{\prime} 12,5^{\prime \prime} \mathrm{W}$, altitude em relação ao nível do mar de $450 \mathrm{~m}$, ponte com as seguintes dimensões: largura de 3,95 m, comprimento de 7,00 m e vão livre de $6,10 \mathrm{~m}$.

296 Mastela, L. da C.; Segundinho, P. G. de A.; Taquetti, V. B.; Christoforo, A. L.; Lahr, F. A. R.; Gales, T. A. 
A execução da ponte Florestinha foi realizada empregando-se 12 vigas de madeira da espécie Eucalyptus citriodora. Durante a inspeção, verificou-se que as peças tinham teor de umidade médio de $20 \%$. As dimensões dos elementos estruturais foram de $700 \mathrm{~cm}$ de comprimento, e seção circular de diâmetro médio no centro do vão igual a $30 \mathrm{~cm}$. Os valores médios de resistência e módulo de elasticidade à flexão adotados são fc0,m igual a $50 \mathrm{MPa}$, ft0,m igual a 99,7 $\mathrm{MPa}$ e $\mathrm{E}_{\mathrm{m}}$ igual a 14.292,16 $\mathrm{MPa}$, que correspondem aos valores apresentados na NBR 7190 (ABNT, 1997) quando corrigidos.

O tabuleiro foi executado em concreto com $12 \mathrm{~cm}$ de altura média, $700 \mathrm{~cm}$ de comprimento e $395 \mathrm{~cm}$ de largura. Foi utilizado concreto de resistência característica à compressão $\left(\mathrm{f}_{\mathrm{ck}}\right)$ igual a $18 \mathrm{MPa}$, moldado in loco, com módulo de elasticidade $\left(\mathrm{E}_{\mathrm{c}}\right)$ estimado aos 28 dias, em 23.758,8 $\mathrm{MPa}$ e módulo de elasticidade secante $\left(\mathrm{E}_{\mathrm{cs}}\right)$ de $20.200 \mathrm{MPa}$. O módulo de elasticidade transversal $\left(\mathrm{G}_{\mathrm{c}}\right)$ foi adotado como $8.080 \mathrm{MPa}$.

As principais características da ponte Florestinha são apresentadas na Figura 2(a), que mostra a seção transversal utilizada no estudo. Um veículo-tipo foi empregado para executar as provas de cargas. Neste caso, utilizou-se um caminhão com dois eixos traseiros, e adotaram-se carregamentos de $120 \mathrm{kN}$ por eixo. O trem-tipo foi posicionado sobre o tabuleiro centrado (denominado central) e deslocado lateralmente (denominado lateral) em relação ao eixo longitudinal da laje de concreto, de acordo com a Figura 2(b).

Conectores em "X" de barras de aço CA50 com diâmetro igual a $8 \mathrm{~mm}$, resistência média no estado limite último de $30 \mathrm{kN}$ e módulo de deslizamento médio de $38 \mathrm{kN} / \mathrm{mm}$ foram adotados nas ligações entre a laje de concreto e a viga de madeira. Os conectores possuem inclinação de $45^{\circ} \mathrm{em}$ relação ao eixo das vigas. $\mathrm{Na}$ Figura 2(c), podem-se observar os espaçamentos entre os conectores.

A modelagem numérica com a aplicação do método dos elementos finitos foi feita usando-se como base os dados disponibilizados no trabalho de Pigozzo (2004), por meio de ensaios e pelo programa de cálculo estrutural Orthotropic Timber Bridges (OTB). Este programa foi desenvolvido para a aplicação em pontes mistas de madeira e concreto e pontes protendidas, as quais podem apresentar seção simples, seção $\mathrm{T}$, seção caixão e treliçada. O programa OTB utiliza a solução por séries para resolver a equação de placas ortotrópicas. Nesse caso, ocorre a geração automática dos pontos dos resultados, os quais são baseados em uma malha inserida pelo usuário, a qual tem como função definir os pontos para os quais serão apresentados os resultados, e sua discretização não interfere nos resultados.

O programa Structural Analysis Program (SAP2000) estudante foi empregado para a obtenção dos deslocamentos verticais. Três modelos foram utilizados para a modelagem numérica do tabuleiro misto constituído de madeira e concreto. Cada modelo apresenta dois tipos de carregamento, o carregamento central e o carregamento lateral. A diferença entre os modelos 1 e 2 está nos conectores entre a viga e o tabuleiro.

No modelo 3, as vigas foram consideradas como sendo de madeira laminada colada (MLC). O uso de MLC é justificado por ser a forma mais adequada de vencer grandes vãos, visto que é uma tecnologia desenvolvida para esse fim. Além disso, com a crescente preocupação com o meio ambiente e a exigência de medidas construtivas sustentáveis, a MLC é uma alternativa viável por permitir maior aproveitamento do material e uso de madeira reflorestada. Por ser um produto industrializado, é possível controlar as propriedades mecânicas, como o módulo de elasticidade.

\section{Figura 1 - Ponte Florestinha}
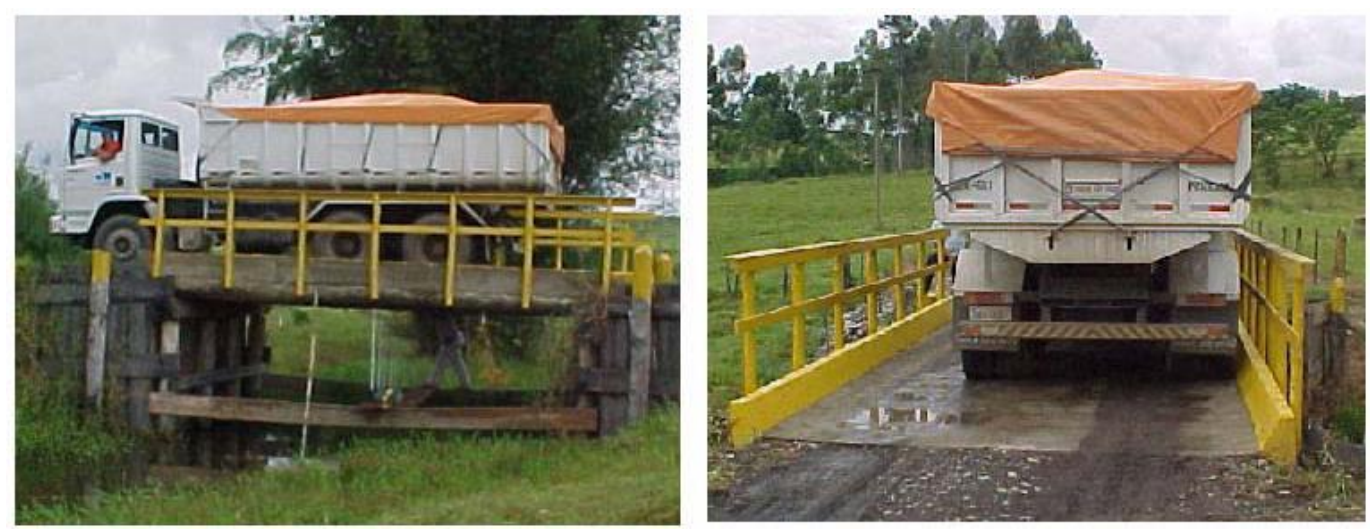

Fonte: Pigozzo (2004). 
Figura 2 - Características da ponte Florestinha

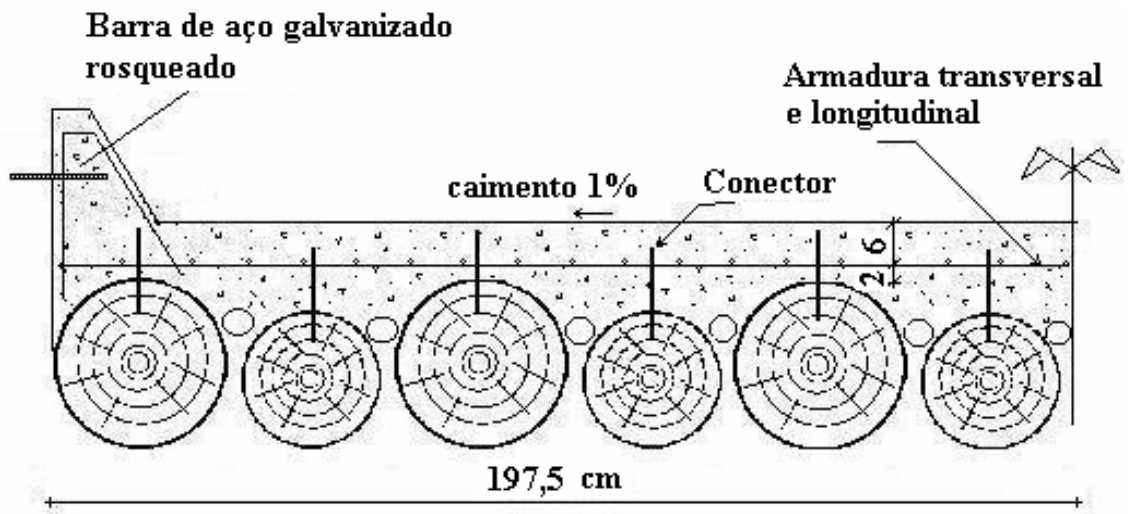

(a) Seção transversal adotada
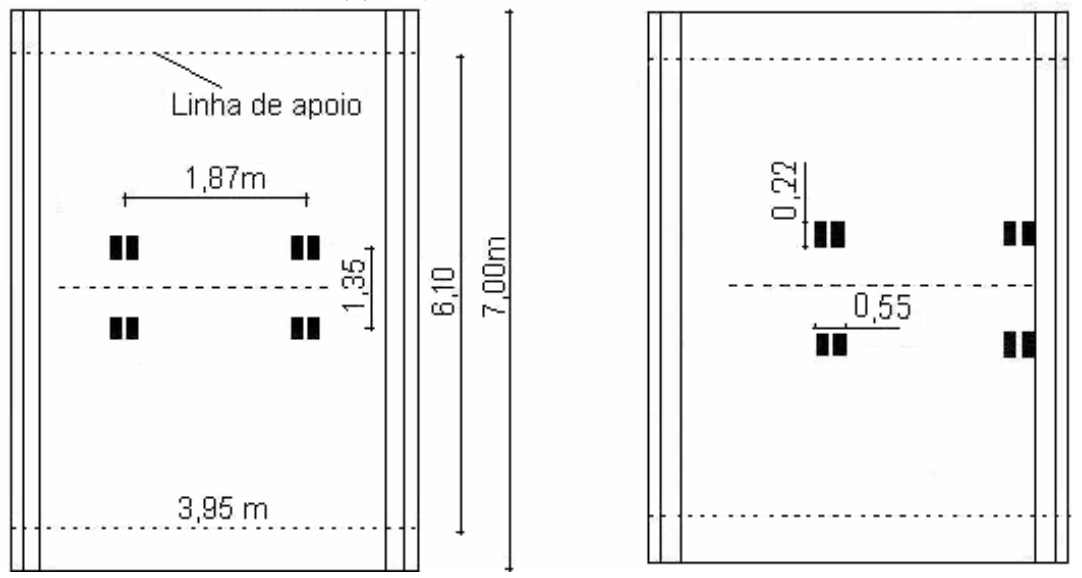

(b) Posicionamento do trem-tipo sobre o tabuleiro, carregamento 1 centrado, e carregamento 2 na lateral

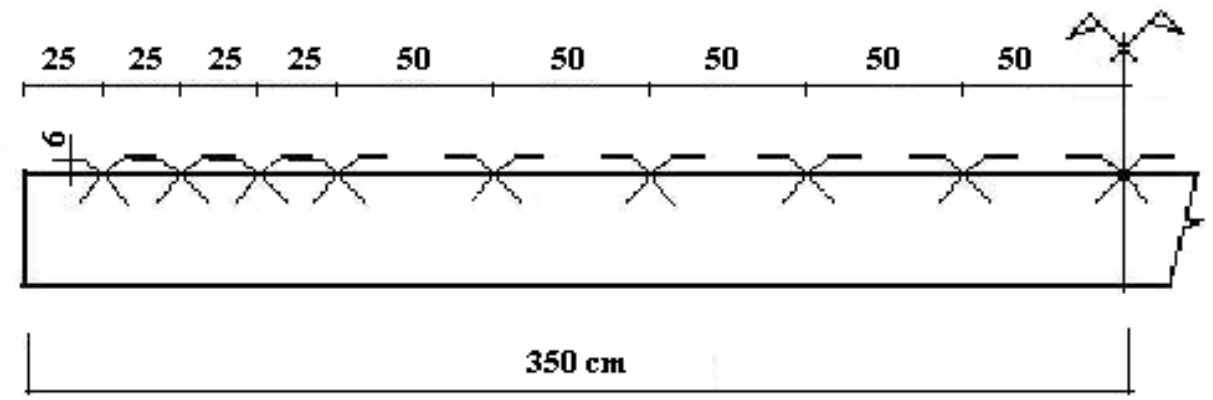

(c) Disposição dos conectores "X" nas vigas de madeira

Fonte: Pigozzo (2004).

Os conectores dos modelos 1 e 3 estão no sentido perpendicular ao elemento estrutural. O modelo 2 possui ligações entre nós e não utiliza conectores. A Figura 3 apresenta a vista em três dimensões da parte inferior do tabuleiro de madeira e concreto para os três modelos gerados no SAP2000.

Algumas adaptações foram feitas nos modelos numéricos para que os resultados das discretizações dos elementos que compõem as vigas mistas representassem o desempenho estrutural verificado experimentalmente.

Para tanto, foi necessário restringir as translações dos nós situados nos apoios existentes nas extremidades das vigas nas três direções. Além disso, foi necessário liberar as rotações dos eixos principais. O espaçamento adotado entre as vigas foi de $33 \mathrm{~cm}$. A Figura 4(a) apresenta a vista frontal do tabuleiro misto e as translações dos nós que ocorrem nas vigas de madeira, representadas nos modelos 1 e 2 . A Figura 4(b), gerada no programa SAP2000, apresenta a vista frontal do tabuleiro misto do modelo 3.

298 Mastela, L. da C.; Segundinho, P. G. de A.; Taquetti, V. B.; Christoforo, A. L.; Lahr, F. A. R.; Gales, T. A. 
Figura 3 - Vista em três dimensões da parte inferior do tabuleiro de madeira e concreto

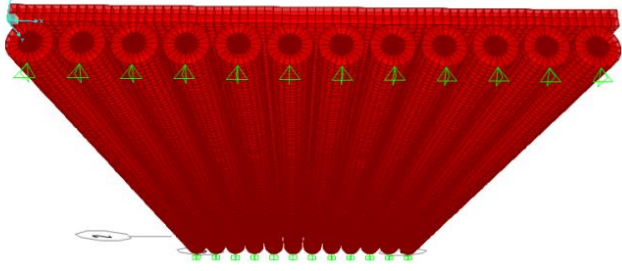

(a) Para os modelos 1 e 2

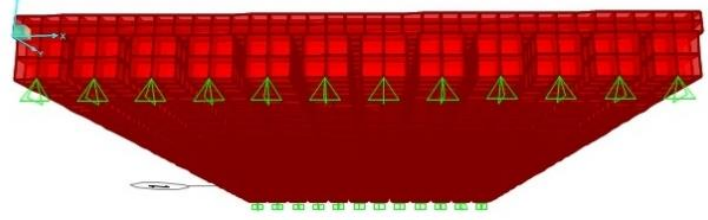

(b) Para o modelo 3

Figura 4 - Vista frontal do tabuleiro de madeira e concreto e as translações dos nós dos apoios nas extremidades das vigas de madeira

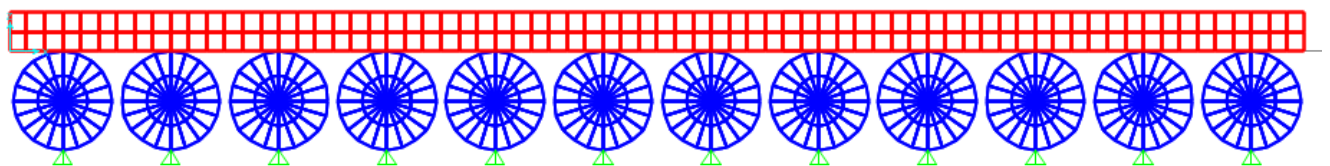

(a) Modelos 1 e 2



(b) Modelo 3

Os coeficientes de Poisson empregados para o concreto e para a madeira foram de 0,20 e de 0,30 respectivamente. Na modelagem, considerou-se a madeira como um material isotrópico, apesar de sua característica ortotrópica. Em todos os modelos numéricos propostos, a laje de concreto foi discretizada em 5.460 elementos de sólidos.

$\mathrm{Na}$ simulação, aplicaram-se as cargas nos nós onde se localizam os sólidos da laje, que são os mesmos pontos onde foram aplicadas as cargas no ensaio feito em campo. A origem do sistema de coordenadas cartesianas foi considerada na parte inferior do tabuleiro, conforme pode ser observado nas Figura 3(a) e 3(b). Os pontos cartesianos do tabuleiro para o carregamento central foram A $(104 ; 280 ; 0)$, B (291; 280; 0), C $(291 ; 410 ; 0)$ e D $(104 ; 410 ; 0)$. No caso do carregamento lateral, os pontos adotados foram A $(148 ; 280$; $0)$, B $(335 ; 280 ; 0), \mathrm{C}(335 ; 410 ; 0)$ e D $(148 ; 410 ; 0)$. A carga adotada em todos os pontos é de $60 \mathrm{kN}$, originada do veículo-tipo empregado para os três modelos estudados.

Os modelos 1 e 2, que consideram vigas roliças de diâmetro médio igual a $30 \mathrm{~cm} \mathrm{e} \mathrm{a} 700 \mathrm{~cm}$ de comprimento respectivamente, foram discretizados em 2.800 elementos de sólidos. Cada elemento possui comprimento de $10 \mathrm{~cm}$, utilizando ângulo de $18^{\circ}$ de rotação. No modelo 3 , considerou-se que as vigas possuem seções quadradas, diante do princípio da equivalência da inércia. Dessa forma, as vigas do modelo 3 possuem dimensões igual a $(26 \times 700 \times 26) \mathrm{cm}$, e estas foram discretizadas em 280 elementos de sólido, que possuem dimensões de $(13 \times 10 \times 13) \mathrm{cm}$. Posteriormente à discretização das vigas, realizou-se a verificação dos valores dos nós (joints) no ponto central de cada viga, de forma a se obterem os deslocamentos verticais. Para a discretização eficiente da malha, foi necessária a análise dos modelos numéricos a partir de diferentes níveis de refinamento, até que os resultados levassem a respostas condizentes de deslocamentos, ou seja, quando os erros entre as comparações dos deslocamentos se aproximassem de zero.

A simulação de uma barra do elemento Frame foi feita para representar a conexão entre as vigas de madeira e a laje de concreto, conforme o princípio da equivalência da inércia. Tal adaptação foi necessária tendo em vista que o programa não possibilita a elaboração de modelo de conectores como o adotado na execução da ponte. Dessa forma, a modelagem considerou somente a translação dos nós em relação às barras no sentido perpendicular ao tabuleiro de madeira e concreto, e a rotação em relação ao eixo perpendicular. Nos modelos 1 e 3, foram empregados conectores perpendiculares à viga com comprimento igual a $12 \mathrm{~cm}$ e diâmetro igual a $8 \mathrm{~mm}$. O modelo 2 considerou apenas as ligações por nós.

Para a modelagem numérica utilizando o programa computacional SAP2000, foi utilizado um módulo de elasticidade do concreto secante da madeira e dos pinos conforme apresentado no projeto e na execução da ponte Florestinha (PIGOZZO, 2004). 
Para todos os modelos, adotou-se o módulo de elasticidade médio do concreto como sendo de 20.200 MPa, e o módulo de elasticidade médio da madeira como sendo de 14.292,16 MPa.

Fez-se a análise estatística dos dados obtidos com o objetivo de determinar o valor do erro médio (EM) e do desvio padrão do erro (DPE) para os deslocamentos verticais encontrados nos carregamentos centrais e laterais dos modelos.

Para o EM, Equação 1, tem-se que:

$\mathrm{EM}=\frac{1}{\mathrm{n}} \sum_{\mathrm{i}=1}^{\mathrm{n}}\left|\mathrm{X}_{\text {simulado (i) }}-\mathrm{X}_{\text {medido(i) }}\right|$ Eq. 1

em que:

Xsimulado(i) é o valor simulado na posição i; e

Xmedido(i) é o valor obtido na posição i.

Para o erro percentual médio (EPM), Equação 2, tem-se:

$\mathrm{EPM}=\frac{1}{\mathrm{n}} \sum_{\mathrm{i}=1}^{\mathrm{n}}\left|\mathrm{EP}_{\mathrm{i}}\right|$

Eq. 2

em que $\mathrm{EP}_{\mathrm{i}}=100 \times\left(\frac{\mathrm{E}_{\text {exp }}-\mathrm{E}_{\text {prog }}}{\mathrm{E}_{\exp }}\right)$ são os ganhos em porcentagem para a i-ésima observação $(\mathrm{i}=$ $1,2, \ldots, n)$. Esse valor considera um sinal, indicando se a função subestima $(+)$ ou superestima (-) o valor observado.

A Equação 3 pode ser escrita da mesma forma que a Equação 2:

$\mathrm{EPM}=\frac{100}{\mathrm{n}} \sum_{\mathrm{i}=1}^{\mathrm{n}}\left|\frac{\mathrm{E}_{\text {exp }}-\mathrm{E}_{\text {prog }}}{\mathrm{E}_{\exp }}\right|$

O DPE é apresentado na Equação 4:

$\mathrm{DPE}=\sqrt{\frac{\frac{1}{\mathrm{n}} \sum_{\mathrm{i}=1}^{\mathrm{n}}\left(\mathrm{x}_{\text {erro }(\mathrm{i})}-\overline{\mathrm{x}}\right)^{2}}{\mathrm{n}-1}}$

em que:

Xerro(i) é o valor do erro na posição i;

$\overline{\mathrm{X}}$ é a média dos valores do erro $\mathrm{i}$;

n é o número de valores da amostra; e

$\mathrm{i}$ indica o índice que determina a posição simulada.

Estatística descritiva foi empregada para a análise de média e de coeficiente de variação (CV).

\section{Resultados e discussões}

Os valores dos deslocamentos verticais foram obtidos utilizando-se os valores experimentais e o programa OTB de acordo com Pigozzo (2004), e o método dos elementos finitos, em que se fez a simulação dos modelos 1, 2 e 3 . As Figuras 5(a) e 5(b) permitem comparar os dados experimentais, OTB e o MEF para o carregamento central e para o carregamento lateral respectivamente.

As Tabelas 1 e 2 apresentam a análise estatística para o carregamento central e para o carregamento lateral respectivamente, o que permite a comparação dos modelos com o modelo experimental.

Observa-se que os resultados encontrados para o modelo 1, pelo método de elementos finitos, estão próximos aos resultados experimentais, conforme apresentado nas Figuras 5(a) e 5(b). No modelo 1, as ligações são constituídas por elementos de barra (frame) cujas ligações são rígidas, tanto na madeira quanto no concreto, isto é, apresentam restrições de movimento. Quando se compara o modelo 2 aos resultados obtidos no OTB, nota-se que o modelo 2 apresentou melhores resultados. Entretanto, o modelo 2 ofereceu resultados pouco significativos em comparação ao modelo 1, que emprega conectores perpendiculares à viga, conforme executado na ponte estudada. Embora o modelo 2 tenha apresentado erro percentual médio baixo quando comparado ao modelo experimental, observa-se que aquele apresenta discrepância em relação ao resultado experimental. 
Figura 5 - Comparação entre os modelos dos deslocamentos verticais

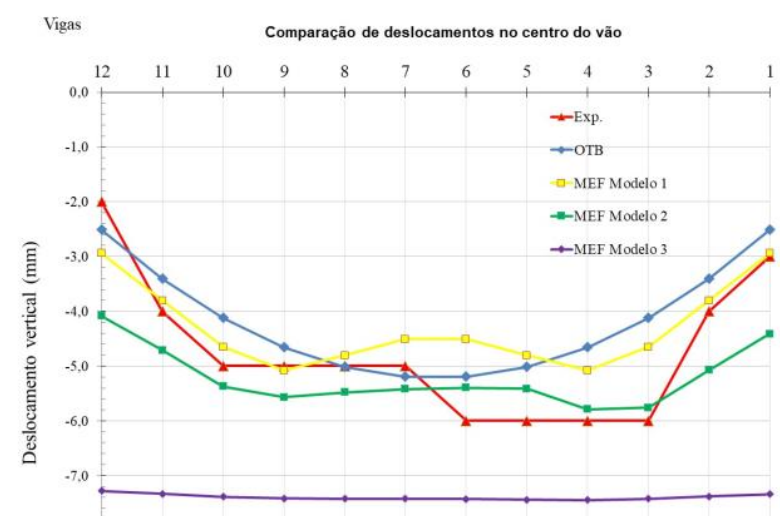

(a) Na seção transversal central com o carregamento central

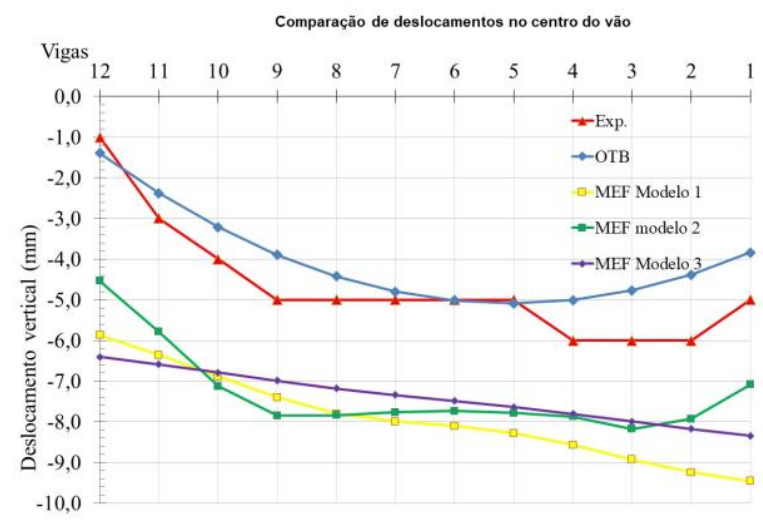

(b) Na seção transversal central com o carregamento lateral

Tabela 1 - Valores para o carregamento central

\begin{tabular}{l|c|c|c|c}
\hline & OTB & MEF 1 & MEF 2 & MEF 3 \\
\hline Erro médio (mm) & 2,42 & 0,45 & 0,46 & 2,64 \\
Erro percentual médio (\%) & 1,04 & 1,56 & 1,09 & 0,94 \\
Desvio padrão (mm) & 1,32 & 0,69 & 0,81 & 1,24 \\
Coeficiente de variação (\%) & 0,54 & 1,53 & 1,77 & 0,46 \\
\hline
\end{tabular}

Tabela 2 - Valores para o carregamento lateral

\begin{tabular}{l|c|c|c|c}
\hline & OTB & MEF 1 & MEF 2 & MEF 3 \\
\hline Erro médio (mm) & 0,94 & 3,24 & 2,62 & 2,73 \\
Erro percentual médio (\%) & 0,25 & 1,12 & 0,56 & 0,65 \\
Desvio padrão (mm) & 1,49 & 0,73 & 0,51 & 1,00 \\
Coeficiente de variação (\%) & 1,58 & 0,22 & 0,19 & 0,36 \\
\hline
\end{tabular}

Deve-se observar que os dados experimentais sofrem variação devido à sensibilidade do equipamento utilizado em campo. Apesar disso, a ordem de grandeza dos resultados permite uma previsão satisfatória do comportamento real (PIGOZZO et al., 2018).

A comparação entre o modelo 3 e o experimental mostrou que aquele é o que possui pior representatividade entre os modelos estudados. Além disso, o modelo 3 apresentou maiores deslocamentos no vão central do que os demais modelos. Dessa forma, esse modelo ocasiona a majoração dos resultados do deslocamento. $\mathrm{O}$ modelo 3 apresentou ainda uma seção quadrada, que foi tomada como uma viga de MLC com equivalência de inércia na direção vertical à seção circular. No entanto, o comportamento global do sistema considerando que ocorreram rotações não apresentou resultados satisfatórios. Acredita-se que a malha da viga de MLC foi 
cerca de 3 vezes menos refinada do que a das vigas circulares de madeira. Logo, o material de MLC não foi determinante na obtenção dos resultados.

Segundo Oudjene et al. (2018), a representação de conectores em simulações numéricas apresenta limitações, tendo em vista as escalas aplicadas aos modelos de elementos finitos de grandes dimensões e a quantidade de conectores que possuem diâmetro relativamente pequenos.

A pesquisa de Forti et al. (2015) também foi realizada com vigas mistas de madeira e concreto, utilizando o método dos elementos finitos para comparar seus resultados com resultados numéricos obtidos por Forti (2004) e com ensaios de laboratório de Soriano (2001). Segundo Forti et al. (2015), os resultados numéricos foram semelhantes aos resultados experimentais. Nos estágios iniciais, os valores obtidos apresentaram diferenças menores que $10 \%$, confirmando que o método dos elementos finitos é aplicável para diferentes composições estruturais. Os resultados obtidos no presente estudo também mostram a eficiência na aplicação do método dos elementos finitos.

\section{Considerações finais}

A partir dos resultados obtidos, constatou-se que o modelo 1 se aproxima dos dados experimentais e apresenta desvio padrão do erro para o carregamento central de $\pm 0,69 \mathrm{~mm}$, que corresponde a $1,56 \%$ do erro percentual médio; e para o carregamento lateral, de $\pm 0,73 \mathrm{~mm}$, que indica $1,12 \%$ do erro percentual médio.

De acordo com os resultados encontrados por meio do método dos elementos finitos, é possível constatar que o modelo 1 apresenta valores que se aproximam dos resultados experimentais. Logo, esse modelo numérico é capaz de representar os esforços da estrutura mista avaliada com uma boa aproximação.

Os modelos 2 e 3 apresentaram resultados pouco significativos em comparação ao modelo 1. Apesar disso, o modelo 2 apresentou erro percentual médio baixo quando comparado ao modelo experimental. O modelo 3 não apresentou resultados satisfatórios. Para aprimorar o modelo, seria necessário melhorar o refinamento da malha.

Os modelos numéricos 1 e 2 usados representaram satisfatoriamente os modelos experimentais, revelando que a aplicação do método dos elementos finitos foi satisfatória para a análise da ponte em questão.

\section{Referências}

ASSOCIAÇÃO BRASILEIRA DE NORMAS TÉCNICAS. NBR 7190: Projeto de estruturas de madeira. Rio de Janeiro, 1997.

AZEVEDO, A. F. M. Método dos elementos finitos. 2003. Disponível em:

http://www.alvaroazevedo.com/publications/books/livro_mef_aa_1ed/doc/Livro_MEF_AA.pdf. Acesso em: 02 out. 2019.

CALIL JUNIOR, C.; DIAS, A. A. Utilização da madeira em construções rurais. Revista Brasileira de Engenharia Agrícola e Ambiental, v. 1, p. 71-77, 1997.

COMPUTERS AND STRUCTURES. SAP2000: integrated finite element analysis and design of structures, version 20 Ultimate Netwoork, software. Berkeley, 2012.

DIAS, A. et al. Timber-concrete-composites increasing the use of timber in construction. European Journal of Wood Products, v. 74, n. 3, p. 443-451, 2015.

FORTI, N. C. S. Análise numérica de vigas mistas em concreto e madeira. 2004. Tese (Mestrado em Engenharia Civil) - Faculdade de Engenharia Civil, Universidade Estadual de Campinas, Campinas, 2004.

FORTI, N. C. S. et al. Análise de vigas mistas de concreto e madeira pelo método dos elementos finitos.

Revista Ibracon de Estruturas e Materiais, v. 8, n. 4, p. 507-528, 2015.

FRANGIACOMO, M. A finite element model for long-term analysis of timber-concrete composite beams. Structural Engineering and Mechanics, v. 20, n. 2, p. 173-189, 2005.

LOTTI, R. S. et al. Aplicabilidade científica do método dos elementos finitos. Revista Dental Press de Ortodontia e Ortopedia Facial, v. 11, n. 2, p. 35-43, 2006.

MASCIA, N. T. et al. Study of concrete-timber composite beams using an analytical approach based on the principle of virtual work and experimental results. Engineering Structures, v. 46, p. 302-310, 2013. 
MOLINA, J. C.; CALIL JUNIOR, C. Estratégia para modelagem numérica de sistemas mistos considerando diferentes modelos de ruptura para os materiais. Revista Chilena de Ingeniería, v. 17, n. 2, p. 256-266, 2009.

MOLINA, J. C.; CALIL JUNIOR, C. Sistema misto de madeira e concreto: uma abordagem numérico e experimental para altas temperaturas. Matéria, v. 23, n. 3, 2018.

MONTEIRO, S. R. S.; DIAS, A. M. P. G.; LOPES, S. M. R. Bi-dimensional numerical modeling of timberconcrete slab-type structures. Materials and Structures, v. 48, p. 3391-3406, 2015.

OUDJENE, M. et al. Finite element modelling of the nonlinear load-slip behaviour of full-scale timber-toconcrete composite T-shaped beams. Composite Structures, v. 196, p. 117-126, 2018.

OUDJENE, M. et al. Non-linear finite element modelling of the structural behaviour of screwed timber-toconcrete composite connections. Composite Structures, v. 102, p. 20-28, 2013.

PIGOZZO, J. C. Estudos e aplicações de barras de aço coladas, como conectores em placas mistas de madeira e concreto para tabuleiros de pontes. 2004. Tese (Doutorado em Engenharia de Estruturas) Universidade Estadual Paulista, São Carlos, 2004.

PIGOZZO, J. C. et al. Design and execution of wood-concrete deck bridge. Current Journal of Applied Science and Technology, v. 28, p. 1-10, 2018.

RODRIGUES, J. N.; DIAS, A. M. P. G.; PROVIDÊNCIA, P. Timber-concrete composite bridges: state-ofthe-art review. Bio Resources, v. 8, n. 4, p. 6630-6649, 2013.

SEGUNDINHO, P. G. A. Análise teórico-experimental de um tabuleiro misto madeira-concreto composto por vigas circulares. 2005. Dissertação (Mestrado em Engenharia Civil) - Faculdade de Engenharia de Ilha Solteira, Universidade Estadual Paulista, Ilha Solteira, 2005.

SORIANO, J. Estruturas mistas de concreto e madeira: análise de vigas e painéis e aplicações na construção civil. 2001. Tese (Doutorado em Engenharia Agrícola) - Faculdade de Engenharia Agrícola, Universidade Estadual de Campinas, Campinas, 2001.

YEOH, D. et al. State of the art on timber-concrete composite structures literature review. Journal of Structural Engineering, v. 137, n. 10, p. 1085-1095, 2011.

ZONA, A.; BARBATO, M.; FRAGIACOMO, M. Finite-element model updating and probabilistic analysis of timber-concrete composite beams. Journal of Structural Engineering, v. 138, n. 7, p. 899-910, 2012.

\section{Agradecimentos}

Ao Programa de Pós-Graduação em Ciências Florestais da Universidade Federal do Espírito Santo e ao Conselho Nacional de Desenvolvimento Científico e Tecnológico ( $\mathrm{CNPq}$ ) pelos recursos disponibilizados para a realização da pesquisa. 


\author{
Leonor da Cunha Mastela \\ Departamento de Ciências Forestais e Madeira | Universidade Federal do Espírito Santo | Av. Gov. Lindemberg, 316, Centro | Jerônimo \\ Monteiro - ES - Brasil | CEP 29500-000 | Tel.: (28) 3538-2515 | E-mail: leomastela@yahoo.com.br

\section{Pedro Gutember de Alcântara Segundinho} \\ Departamento de Ciências Forestais e Madeira | Universidade Federal do Espírito Santo | E-mail: p_gutemberg2001@yahoo.com.br

\section{Vinicius Borges Taquetti} \\ Departamento de Ciências Forestais e Madeira | Universidade Federal do Espírito Santo | E-mail: viniciustaquetti@hotmail.com

\section{André Luis Christoforo} \\ Departamento de Engenharia Civil | Universidade Federal de São Carlos | Rodovia Washington Luís, km 235 | São Carlos - SP - Brasil | \\ CEP 36307-352 | Tel.: (16) 3351-8262 | E-mail: alchristoforo@ufscar.br

\section{Francisco Antonio Rocco Lahr} \\ Escola de Engenharia de São Carlos | Universidade Federal de São Carlos | Av. Trabalhador Sãocarlense, 400 | São Carlos - SP - Brasil | \\ CEP 13566-590 | Tel.: (16) 3373-9455 | E-mail: frocco@sc.usp.br

\section{Tiago Aguiar Gales} \\ Departamento de Ciências Forestais e Madeira | Universidade Federal do Espírito Santo | E-mail: tgales44@gmail.com
}

\title{
Ambiente Construído
}

Revista da Associação Nacional de Tecnologia do Ambiente Construído

Av. Osvaldo Aranha, $99-3^{\circ}$ andar, Centro

Porto Alegre - RS - Brasil

$$
\text { CEP } 90035-190
$$

Telefone: +55 (51) 3308-4084

www.seer.ufrgs.br/ambienteconstruido www.scielo.br/ac

E-mail: ambienteconstruido@ufrgs.br

(c) () 